\title{
Energy Conversion Monitoring in Skin Tissue: A More Sensitive Measure of Hypoxia Occurring With Sleep Disordered Breathing Than Pulse Oximetry
}

Guy M. Hatch, MD ${ }^{1}$, Liza Ashbrook, MD², Aric A. Prather, Ph.D², Andrew D. Krystal, MD, MS²

${ }^{1}$ Reveal Biosensors, Inc, Logan, Utah, USA

2University of California San Francisco, San Francisco, CA, USA

Corresponding Author:

Andrew D. Krystal, MD, MS

401 Parnassus Ave

San Francisco, CA USA 94143

andrew.krystal@ucsf.edu

Authorship Statement: All authors have seen and approved this manuscript

Author Declarations:

Guy Hatch: Financial Support-None; Off-label/Investigational Use - Energy Conversion Monitoring is not FDA approved and is investigational. Conflict of Interest - Co-Founder and Chief Technology Officer of Reveal Biosensors, Inc.

Liza Ashbrook: Financial Support-None; Off-label/Investigational Use - Energy Conversion Monitoring is not FDA approved and is investigational. Conflict of Interest None.

Aric Prather: Financial Support-Research Support from NIH, Headspace, Inc; Offlabel/Investigational Use - Energy Conversion Monitoring is not FDA approved and is investigational. Conflict of Interest - None.

Andrew Krystal: Financial Support-Research Support from Reveal Biosensors; Offlabel/Investigational Use - Energy Conversion Monitoring is not FDA approved and is investigational. Conflict of Interest-Research Support from NIH, Janssen, Jazz. Axsome, Reveal Biosensors; Consultant to Adare, Eisai, Ferring, Galderma, Harmony Biosciences, Idorsia, Jazz, Janssen, Takeda, Merck, Neurocrine, Pernix, Physician's Seal

Number of Tables: 1

Number of Figures: 7

Abstract Word Count: 286 words

Manuscript Word Count: 3761 words 


\section{ABSTRACT}

Pulse oximetry is the current standard for detecting drops in arterial blood oxygen saturation $\left(\mathrm{SpO}_{2}\right)$ associated with obstructive sleep apnea and hypopnea events in polysomnographic (PSG) testing. In cases of hypoxic challenge, such as occurs during apneic events, regulatory mechanisms restrict blood flow to the skin to preferentially maintain $\mathrm{SpO}_{2}$ for more vital organs. As a result, a measure related to skin tissue oxygenation is likely to be more sensitive to inadequate breathing during sleep than pulse oximetry. Energy Conversion Monitoring (ECM) provides a method for measuring skin tissue oxygen-dependent energy conversion and, as such, is promising for more sensitively detecting sleep disordered breathing (SDB) events compared to pulse oximetry. We hypothesized that ECM would detect hypoxia occurring with SDB events associated with drops in $\mathrm{SpO}_{2}$ but also would detect hypoxic challenge occurring with $\mathrm{SDB}$ events not associated with drops in $\mathrm{SpO}_{2}$ (hypopneas defined by a drop in nasal pressure occurring in conjunction with an arousal, respiratory-related arousals, and primary snoring). Primary snoring is of particular interest with respect to the potential of ECM because it is statistically associated with co-morbidities of SDB, such as hypertension, but is not considered pathological because of the lack of a proximal measure of pathology occurring with PSG. In this article we review ECM technology and methodology, present preliminary data indicating that it detects hypoxia occurring in the skin during SDB events that is not detected as blood desaturation by pulse oximetry, and make the case that it is a promising tool for identifying pathology occurring at the mild end of the SDB spectrum.

Keywords-Tissue hypoxia; sleep disordered breathing; primary snoring; energy conversion monitor; tissue hyperoxia 


\section{INTRODUCTION}

The presence of pathology occurring with sleep disordered breathing (SDB) is currently identified during polysomnography (PSG) by the presence of a drop in arterial blood oxygen saturation $\left(\mathrm{SpO}_{2}\right)$, as measured with pulse oximetry, or electroencephalographic (EEG) evidence of sleep disturbance. ${ }^{1-4}$ These findings are used as the basis for the widely employed definitions for apneas, hypopneas, and respiratory related arousals (RERAs), which form the basis of the diagnosis of SDB and determine whether to institute therapy. ${ }^{1,2-}$ 4 Those events without a drop in $\mathrm{SpO}_{2}$ (hypopneas associated only with arousals and RERAs) are presumed to represent challenges to adequate respiration occurring due to obstructive events that do not cause hypoxemia but are pathological because they disturb sleep. Of note, some SDB events at the mild end of the spectrum neither cause drops in $\mathrm{SpO}_{2}$ nor disturb sleep and, as a result, are not considered pathological. An example is primary snoring. ${ }^{1}$

Despite the absence of a proximal measure of pathology occurring during the sleep study (i.e., drop in $\mathrm{SpO}_{2}$ or disturbance of sleep), there is clear evidence that primary snoring is associated with significant medical morbidity indicating that it is indeed a cardiovascular stressor. Primary snoring has long been statistically linked to significantly increased risk of hypertension, ${ }^{5}$ heart disease and stroke, ${ }^{6}$ premature births, ${ }^{7}$ and in children, to developmental delay, attention deficit disorder, and socialization and learning disabilities. ${ }^{8}$ Recent research has also identified two blood pressure-related phenomena linked to snoring; (1) lack of normal blood pressure 'dipping', ${ }^{9}$ and (2) the occurrence of a blood pressure surge following snoring episodes. ${ }^{10}$ 
Despite the epidemiologic evidence for a link to the negative medical outcomes described above, without some objective evidence of pathology proximal to the snoring itself, there is currently no basis for instituting therapy for primary snoring that might prevent the occurrence of these statistically associated long-term morbidities.

Detecting snoring-associated pathology would require a measure that detects a proximal trigger of cardiovascular morbidity with greater sensitivity than current methods. One such possibility would be a measure that is more sensitive to hypoxic stress than pulse oximetry. Such a measure would also have the potential to determine the extent to which hypoxic stress is occurring with hypopneas associated with arousals and RERAs. A new biometric that has such promise is energy conversion monitoring $(E C M)$ in skin tissue.

\section{PULSE OXIMETRY VS. ENERGY CONVERSION MONITORING}

Pulse oximetry, invented in 1972, is the product of the search to find a continuous, noninvasive alternative to periodic sampling and laboratory testing of arterial blood to track the oxygen content of blood during surgical anesthesia and intensive care. This blood oxygen information is clinically useful when dealing with several major diseases and abnormal cardiovascular conditions. The ability of pulse oximetry to continuously and painlessly monitor this vital physiologic information eventually gained favor with intensive care clinicians who were initially reluctant to accept the relatively less precise new method. The key to the successful adoption came with the discovery of new insights about the dynamic nature of blood oxygen status that were not evident with timed, and even somewhat staged, blood-gas sampling during surgical anesthesia and intensive care. Pulse oximetry 
has since become an indispensable tool in numerous areas of medical practice, and is the standard for measuring oxygenation during sleep in PSG.

Pulse oximeters measure the light intensity at the peak and trough of photoplethysmogram (PPG) waveforms at red and infrared wavelengths of light after the light has passed through tissue, such as a fingertip. The less than $2 \%$ of full scale (FS) pulsatile waveforms (AC) are the portions of the light signals that are most affected by the oxygen saturation of hemoglobin in the arterioles within the light path. Ratio, logarithmic, and Fast Fourier Transform (FFT) calculations are currently used to produce $\mathrm{SpO}_{2}$ percentage output data from these AC measurements. However, these calculation methods either do not include, or mathematically dispense with, the remaining about $98 \%$ FS of the red and infrared light signals that are non-pulsating (DC). In contrast, the ECM signals utilize the previously unused $98 \%$, non-pulsating DC components of pulse oximeter signals. The available data suggests that this portion of the signal likely indicates the dynamic status of energy conversion processes within tissue mitochondria. ${ }^{15}$ While further study is needed to fully define the sources of the ECM's photonic signal responses, the experimental evidence so far indicates that that oxygen supply-dependent energy conversion molecular phenomena in skin tissue are involved. ${ }^{16}$

It is important to note that there are several commercial and medical device sensors that are called 'tissue oximeters' by their developers, which claim to measure the oxygen saturation of the blood flowing within tissue. These devices typically use infrared light that can penetrate deeper than the skin and claim to measure the average oxygen saturation of the blood hemoglobin $\left(\mathrm{StO}_{2}\right)$ flowing within the deeper tissues, such as muscle and brain. These are currently used clinically to monitor 'consumption of oxygen' by the tissue 
as indicated by changes of the $\mathrm{StO}_{2}$ value. However, the timed (DC) sampling used by 'tissue oximeters' includes the much more prominent confounding variable spectral absorption by the tissue itself, resulting in uncertainty as to what is being reported. Accurate measurement of the molecular oxygen tension, or oxygen content, within tissue, such as with a Clark electrode or by the Lumee $^{\circledR}$ implant and sensor system (Profusa, Inc.) ${ }^{14}$ respectively, has also been demonstrated but these devices have limitations to practical, routine use. Movement of blood cells within capillaries in the skin is also detectable using Doppler-shifted laser light, as an index of blood perfusion in the skin, which is reduced under hypoxic stress by reflex vasoconstriction. This information may be clinically useful as indices of cardiopulmonary health, of molecular oxygen supply in the blood, and of blood flow within tissue. However, the metabolic status of the tissue relative to the oxygen supply must be assumed. None of these sensors can indicate whether the tissue is receiving less than enough oxygen, just the right amount of oxygen, or too much oxygen.

The Reveal ECM uses once per second timed samples of tissue-diffused and absorbed light intensity at unique wavelengths and calculates its Energy Conversion Index (ECi) output data by subtracting the detected infrared intensity value from the detected red intensity value. This provides a continuous numeric scale, with a central, or zero, position at the awake, non-stressed condition, and extends with a negative trend with decreased skin tissue oxygen supply, and a positive trend with excess skin tissue oxygen supply. A measure of skin tissue energy conversion is likely to be a more sensitive measure of inadequate breathing than pulse oximetry. This is because, in cases of hypoxic challenge, 
such as occurs with SDB, regulatory mechanisms restrict blood flow to the skin to preferentially maintain blood oxygen saturation for more vital organs.

Data is collected with the ECM sensor placed on the mid-upper arm. The wavelengths used were determined by spectroscopic analysis carried out during a series of conditions which affect oxygen supply, some of which are presented below, including: (1) exposing individuals to conditions known to under- or over-supply oxygen to skin tissue, including breathing nitrogen or breathing $100 \%$ oxygen; (2) studying an individual during physical exertion; (3) studying an individual during reperfusion of skin following a period of tourniquet-induced halted blood flow; and (4) studying an individual during return to the previously normal blood oxygen saturation following an the extended period of mild hypoxemia occurring during an airline flight. We present the ECM findings along with concomitant pulse oximetry data obtained during several of these conditions as well as during a night of sleep in an individual known to be a loud snorer as initial evidence of "proof of concept" (POC) for ECM.

\section{INITIAL PROOF OF CONCEPT TESTING}

Figures 1-3 illustrate the relationship between the response of ECM and the response of a medical-grade pulse oximeter sensor relative to inhalation of nitrogen vs. inhalation of oxygen, and during and following physical exercise, as the basis of our belief that the energy conversion monitor is detecting decreased and increased skin tissue oxygen supply.

Condition 1. Inhalation of $100 \% \mathrm{~N}_{2}$. When much less than the needed quantity of oxygen is being delivered to skin tissue, as induced experimentally by briefly breathing 
nitrogen gas, the ECM sensor detects increased absorption of the red wavelength of sensor light, with slight decrease, or no change, in the absorption of the infrared light. This leads to a drop in the ECi as can be seen readily in Figure 1. The return to baseline levels of red wavelength absorption immediately follows relief of decreased skin tissue oxygen supply by again breathing air.

The variations in amplitude of the infrared signal are seen in real-time to correspond with breath cycles and offer an analog of the effort of each breath. Real-time observation shows that this signal response is apparently due to breathing-induced variation in the venous blood volume in the skin of the upper arm within the sensor's light path. This response appears to be an optically detected analog of the blood flow basis of pulsus paradoxus and suggests that the infrared signal could serve as a measure of breathing effort during sleep studies.

Condition 2. Inhalation of $100 \% \mathrm{O}_{2}$. On the other hand, if excess oxygen is delivered to skin tissue, as induced by breathing $100 \%$ oxygen, the ECM sensor detects increased absorption of the sensor's infrared wavelength, with a slight decrease in the absorption of the red light (See Figure 2). Both of these photonic signal responses become evident within 5-10 seconds of the onset of changes in the partial pressure of oxygen $\left(\mathrm{ppO}_{2}\right)$ of the breathing gas. Due to extended averaging to suppress motion artifact, pulse oximeter data response may be delayed up to 45 seconds, as shown in Figures 1 and 2 . The lack of data processing delay in the ECi data compared with pulse oximetry data is likely to be of value in a number of potential applications. Remarkably, prolonged exposure to excess oxygen results in progressively increased absorbance of the infrared light as manifested in an increase in $\mathrm{ECi}$; this is indicative of excess supply of oxygen to skin tissue. This 
photonic response is also prolonged for up to several hours after the excess oxygen exposure is discontinued and the subject has returned to breathing room air. Pulse oximeters and 'tissue oximeters' cannot detect increased oxygen supply to tissue.

Condition 3. Physical Exertion. Figure 3 presents data recorded simultaneously during a stationary bicycle interval exercise session. The healthy adult athlete's resting heart rate is in the upper 40 to low 50 beats per minute range. His maximum heart rate is about 170 beats per minute during short bursts of maximum exertion. His $\mathrm{SpO}_{2}$ is typically above $95 \%$ at his living altitude of about 4200 feet above mean sea level (AMSL) and, as the $\mathrm{SpO}_{2}$ graph shows, does not vary significantly during exercise. During his warmup, however, the ECM detects an immediate decrease in skin tissue oxygen supply, likely due to vasoconstriction as a first-line defense against hypotension and hypoxemia as muscle blood flow and oxygen consumption increases. The ECM detects this vasoconstriction in the skin by the reduced absorption of light from less blood in the sensor light path mostly affecting infrared absorption. During the successive three similar intervals of high exertion, the skin tissue oxygen supply decreases significantly. However, there is a progressive lessening of the photonic responses with successive intervals of near-equal physical effort. Upon stopping exercise immediately following the third interval, the ECM indicates that his skin tissue oxygen supply becomes excessive, with relatively less absorption of the red wavelength and increased absorption of the infrared, despite the fact that the blood volume in the sensor light path (infrared trace of the Raw Data) immediately returns to its baseline value range. This hyperoxia response suggests the skin tissue's adaptive response to the decreased available oxygen, due to vasoconstriction, during exercise. 


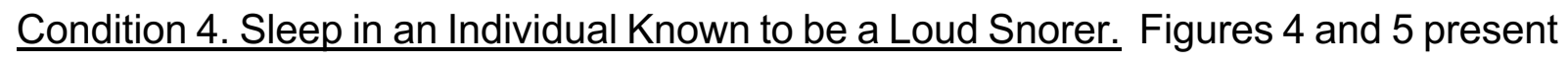
data from a single 9-hour standard clinical PSG carried out in an individual known to be a loud snorer. Figure 4 depicts the ECi trend for the entire night as well as for a selected 10-minute period chosen to illustrate a period where the subject was asleep, snoring, and without change in $\mathrm{SpO}_{2}$, but with a declining $\mathrm{ECi}$ trend. Figure 4 also includes a 1 minute period for which raw PSG data appear in Figure 5 in which this subject is asleep and snoring loudly, is without evidence of blood oxygen desaturation based on pulse oximetry, and where there is only minimal arousal and no evidence of apnea, hypopnea or hypoventilation; and yet, there is a clear decreasing trend in ECi. It should be noted that this selected segment is only one of several such ECi deviations in this study. This recording session also revealed several prolonged periods of decreased skin tissue oxygen supply which were not associated with decreased $\mathrm{SpO}_{2}$.

Results of these initial experiments with the ECM and parallel pulse oximetry provide preliminary evidence that ECM provides an indicator of hypoxia, as reflected with pulse oximetry, but under certain conditions differs from the $\mathrm{SpO}_{2}$ indication of arterial blood oxygen saturation. This dissociation between pulse oximetry and energy conversion monitoring has been found to arise in some settings associated with pathology (breathing $100 \%$ nitrogen), others without pathology (exercise), and in an instance of likely, but not established, pathology (primary snoring). The difference appears to be a function of context. Normal adaptive stress responses, such as reduction in skin perfusion during moderate exertion, are not pathologic and may be an integral part of the health benefits of exercise. However, we hypothesize that if this occurs during sleep, when there is normally a drop in sympathetic tone, it may be harmful. The data recorded during PSG 
from one patient known to be a loud snorer establishes that it is possible to record ECM data during sleep and suggests the potential that ECM might be a more sensitive measure of hypoxic challenge occurring during sleep than pulse oximetry, possibly detecting skin hypoxia occurring during primary snoring, which could be a proximal indicator of cardiovascular stress occurring with this condition.

To further test this hypothesis and provide additional information with respect to establishing POC that ECM might be a more sensitive measure of hypoxic challenge occurring during sleep than pulse oximetry, we carried out a small pilot study of ECM data obtained during PSG.

\section{PILOT PROOF OF CONCEPT STUDY METHODS}

We carried out a pilot study where we obtained ECM data simultaneously with PSG data in a convenience sample of 8 unselected individuals referred for PSG in the UCSF Sleep Disorders Center as part of their clinical management.

Subjects. The subjects consisted of 8 patients all referred for PSG for suspected obstructive sleep apnea. The sample consisted of all those individuals who were willing to sign informed consent for participation during the period of recruitment $(8 / 8 / 2018$ $10 / 17 / 2018)$. Subject age ranged from $31-73$ years of age with a mean age of 52.5 years (standard deviation $=16.3$ years $)$. Subjects were all men except for 1 woman. Subjects were paid $\$ 50$ for participation in the study.

\section{Procedures.}

Subjects were approached for participation during the period of the hookup for their clinical PSGs. Those who provided informed consent had a ECM sensor placed on their 
mid-upper arm attached with a watchband like strap. ECM data was collected simultaneously with PSG obtained according to American Academy of Sleep Medicine (AASM) Guidelines. ${ }^{4}$ PSG included the following channels: Left Outer Canthus of the Eye (LOC); Right Outer Canthus of the Eye (ROC); 6 EEG channels referenced to the contralateral mastoid (F3-M2, F4-M1, C3-M2, C4-M1, O1-M2, and O2-M1); 2 Chin EMG Channels; EKG; A Sound Pressure Microphone (Snore); Nasal Pressure (PTAF), Oronasal Thermocouple (Airflow); Inductive Plethysomography Detection of Thoracic and Abdominal Respiratory Effort (Chest and Abdomen); Left and Right Anterior Tibialis EMG (LLeg and RLeg); and Pulse Oximetry (SAO2).

PSG studies were scored according to AASM guidelines. ${ }^{3,4}$ In addition, we scored events based on the ECM data which we referred to as "hypoventilation events." We used a criterion of at least a 10-point drop in ECi to define an event. This was an initial criterion based on qualitative review of the data presented in Figures 1-5 which includes one night of ECM data collected during PSG. This threshold was also based on the observation that decreases in ECi occurring with heavy snoring were always 10 points or more. It was understood that it is necessary to set an initial ballpark threshold for events lacking criteria for significance with the understanding that, if ECM is indeed to have promise for identifying events of clinical interest, in the future it would be necessary to base event definitions on quantitative methods aimed at optimizing sensitivity and specificity for detection of those events. In order to ensure that events were grounded in changes in breathing established as the respiratory basis for defining clinically significant SDB events, we required that a $10 \%$ drop in ECi be accompanied by a drop in peak nasal pressure of 
$\geqslant 30 \%$ of pre-event baseline nasal pressure lasting at least 10 seconds; which is the AASM criteria for a change in breathing defining hypopneas. ${ }^{3}$

Data Analysis. Analysis consisted of tabulating events scored on the basis of ECM and comparing those with events detected by standard methods including apneas, hypopneas, and RERAs.

\section{PILOT PROOF OF CONCEPT STUDY RESULTS}

The results of the study appear in Table 1. The Apnea-Hypopnea Index (AHI) for the 8 study subjects ranged from 0 to 16.2 events/hr indicating that, by chance, the subjects happened to have a relatively low overall severity of SDB. Five of the eight subjects did not have clinically significant SDB by current criteria, having an AHI of less than 5 .

There was evidence that ECM had a very high level of sensitivity for detecting events defined based on the standard AASM scoring criteria. All apnea and hypopnea events occurring in the 8 subjects were accompanied by a drop in ECi except for 1 (See Figure 6 for examples of apneas accompanied by drops in ECi). However, in some of the subjects, a number of events were detected with ECM that were not detected with standard methods.

For four subjects who had $\mathrm{AHI}<1$ the number of hypoventilation events per hour of sleep (Hypoventilation Events Index) was also $<1$ per hour. In one subject who had an AHI $<1$ the Hypoventilation Events Index was 52.1 (Subject 5 in Table 1) indicating a very high rate of events associated with a drop in nasal pressure without an arousal or blood oxygen desaturation, which were accompanied by a drop in ECi (See Figure 7 for examples of such events). For all 3 subjects who had clinically significant SDB (AHI of 11.8, 16.2, and 
8.4) there was a greater rate of hypoventilation events than scorable apneas and hypopneas. For subject 6 in Table 1 the AHI indicated 11.8 scorable events per hour of sleep while there were 37.1 hypoventilation events per hour of sleep. Because all of the apneas and hypopneas were detected with ECM, this indicates that there were 25.3 hypoventilation events per hour not meeting standard criteria for apneas or hypopneas. For subject 7 in Table 1 the $\mathrm{AHI}$ was 16.2/hr while there were 19 hypoventilation events per hour not meeting apnea or hypopnea criteria. Lastly, subject 8 had an AHI of $8.4 / \mathrm{hr}$ and 22.2 hypoventilation events per hour not meeting apnea or hypopnea criteria.

In terms of safety/tolerability, there were no adverse events of recording ECM in this study and no complaints regarding use of this device.

\section{DISCUSSION}

The initial POC testing carried out establishes basic POC that ECM is sensitive to changes in oxygen supply to skin tissue. ECM reflects decreased oxygen supply occurring with breathing $100 \%$ nitrogen and exercise and indicates excess oxygen supply occurring with breathing $100 \%$ oxygen and during recovery from exertion. Further, initial POC testing suggests that ECM can be recorded and that it detects drops in oxygen intake in an individual who is snoring but not having scorable apnea or hypopnea events.

Our pilot study provides more convincing evidence for POC that ECM might be useful as a measure of the oxygen intake challenge occurring with SDB and might be more sensitive than pulse oximetry for this purpose. Evidence of utility for reflecting SDB is that ECM sensitively detected events associated with pulse oximetry-detected hypoxemia already established to be associated with pathology (apneas and hypopneas). Evidence 
that ECM might be a more sensitive measure of oxygen intake challenge than pulse oximetry is that ECM also detected a change during events established to be associated with pathology where there is no blood oxygen desaturation occurring with pulse oximetry (hypopneas associated with arousals and respiratory related arousals). ECM detected decreased skin tissue oxygen supply occurring in association with events that are not associated with arousals or pulse oximetry-detected blood oxygen desaturation. This suggests the possibility that such events exist on a spectrum of severity with apneas, hypopneas, and RERAs, where the oxygen intake challenge is not sufficient to be detected with pulse oximetry-based blood oxygen measurement, but can be detected with ECM. The greater sensitivity of ECM for these relatively milder oxygen intake challenges is consistent with well-known reflex responses that help maintain blood pressure and minimize decreases in oxygen saturation of the arterial blood supplying the brain and other vital organs at the expense of decreased oxygen supply to the skin. These reflex vascular responses include restricting blood flow to the skin, thus, maintaining blood pressure and prioritizing oxygen availability to vital organs and muscles. ${ }^{12}$

Whether such events are pathological remains to be determined. However, a reason to think they might be pathological is that such events are accompanied by a decrease in nasal pressure, which is already established as the respiratory basis for defining clinically significant events - hypopneas.

The findings also suggest the possibility that decreased skin tissue oxygen supply, as detected by ECM, might be occurring at times in conjunction with snoring even in the absence of decreases in nasal pressure or airflow. This was not directly systematically 
assessed in this study but should be pursued in future work aimed at determining if ECM could serve as a proximal measure of snoring-induced pathology.

It should be noted that, by chance, there was a relatively low overall level of SDB severity in our pilot study sample. To better determine the potential utility of ECM for detecting SDB events it will be important for future work to be carried out including individuals with greater SDB severity.

\section{ABBREVIATIONS:}

AC - Alternating Current

APAP - Auto-titrating Continuous Positive Airway Pressure

COPD - Chronic Obstructive Pulmonary Disease

CSA - Central Sleep Apnea

DC - Direct Current

EEG - Electroencephalogram

FFT - Fast Fourier Transform

FS - Full Scale

$\mathrm{O}_{2}$ - Oxygen

OSA - Obstructive Sleep Apnea

PPG - Photoplethysmogram

PSG - Polysomnogram

SDB - Sleep Disordered Breathing

$\mathrm{SpO}_{2}$ - Arterial Blood Oxygen Saturation

ECi - Energy Conversion Index 
POC- Proof of Concept

Page 17 of 28 
Page 18 of 28 


\section{REFERENCES}

1. American Academy of Sleep Medicine. International Classification of Sleep Disorders. 3rd ed. Darien, IL: American Academy of Sleep Medicine; 2014

2. Smith DL, Gozal D, Hunter SJ, Kheirandish-Gozal L. Frequency of snoring, rather than apneahypopnea index, predicts both cognitive and behavioral problems in young children. Sleep Med. 2017;34:170-178.

3. Berry RB, Budhiraja R, Gottlieb DJ, et al., Rules for scoring respiratory events in sleep: update of the 2007 AASM Manual for the Scoring of Sleep and Associated Events. Deliberations of the Sleep Apnea Definitions Task Force of the American Academy of Sleep Medicine." J Clin Sleep Med. 2012;8(5):597-619.

4. Iber, C., Ancoli-Israel, S., Chesson, A.L.J., and Quan, S.F., "The AASM manual for the scoring of sleep and associated events: rules, terminology and technical specifications." 2007, Westchester, IL: American Academy of Sleep Medicine.

5. Lee, SK, Choi K, Chang YH, et. al., Increased risk of new-onset hypertension in midlife male snorers: The 14-year follow-up study. J Sleep Res. 2018;3:e12757

6. Deeb, R, Smeds MR, Bath J, et. al., Snoring and carotid artery disease: A new risk factor emerges. The Laryngoscope. 2019;129(1):265-268.

7. Dunietz GL, Shedden K, Schisterman EF, et. al., Associations of snoring frequency and intensity in pregnancy with time-to-delivery. Paediatr Perinat Epidemiol, 2018;32(6):504-511.

8. Wada H, Kimura M, Tajima T, et. al. Nocturnal enuresis and sleep disordered breathing in primary school children: Potential implications. Pediatr Pulmonol. 2018;53(11):1541-1548

9. Tamisier R, Weiss JW, Pépin JL, et. al., Sleep biology updates: hemodynamic and autonmic control in sleep disorders. Metabolism Clinical and Experimental. 2018;84:3-10. 
10. Sasaki, N, Nagai M, Mizuno H, et. al., Associations between charactistics of obstructive sleep apnea and nocturnal blood pressure surge. Hypertension. 2018;72(5):1133-1140.

11. Martinez-Garcia, MA, Navarro-Soriano C, Torres G, et. al., Beyond Resistant Hypertension, Hypertension AHA, 2018;72(3):618-624.

12. Kenney WL, Stanhewicz AE, Bruning RS, et. al. Blood pressure regulation III: what happens when one system must serve two masters: temperature and pressure regulation? Eur J Appl Physiol. 2014;114(3): 467-479.

13. Scalzitti, NJ, Sarber KM. Diagnosis and perioperative management in pediatric sleep-disordered breathing. Paediatr Anaesth. 2018;28(11):940-946.

14. Lumee (Profusa, Inc.) Skin tissue oxygen level varies relative to exercise/rest: [https://profusa.com/news-and-events/health-risk-management-value-real-time-monitoringbody-chemistries/]

15. Balu M, Mazhar A, Hayakawa CK, et. al., In vivo multiphoton NADH fluorescence reveals depthdependent keratinocyte metabolism in human skin. Biophysical Journal 2013;104(1):258-67.

16. Hatch, GM, et. al., "Energy Conversion Monitor," Reveal Biosensors, 2018 [https://drive.google.com/open?id=10yqjpOGassmT1PSN6Mx-AEDdz2j4RrTx]. 


\section{Table 1. Pilot POC Study Results}

\begin{tabular}{|c|c|c|c|c|}
\hline Subj & $\begin{array}{c}\text { Apnea Hypopnea } \\
\text { Index }\end{array}$ & $\begin{array}{c}\text { Number of Apneas without Skin } \\
\text { Desaturation }\end{array}$ & $\begin{array}{c}\text { Number of Hypopneas without Skin } \\
\text { Desaturation }\end{array}$ & $\begin{array}{c}\text { Hypoventilation Events } \\
\text { Index }\end{array}$ \\
\hline 1 & 0 & 0 & 0 & 0 \\
0.2 & 0 \\
\hline 2 & 0 & 0 & 0 & 0.3 \\
\hline 3 & 0 & 0 & 0 & 0 \\
\hline 4 & 0 & 0 & 0 & \\
\hline 5 & 0.2 & 1 & 0 & \\
\hline 6 & 11.8 & 0 & 0 & 32.1 \\
\hline 7 & 16.2 & 0 & 0 & 35.1 \\
\hline 8 & 8.4 & 0 & & \\
\end{tabular}

${ }^{*}$ All Indexes Are Events Per Hour of Sleep 
Figure 1. Energy Conversion Index (ECi) recording while breathing nitrogen $\left(\mathrm{N}_{2}\right)$ for 1 minute.

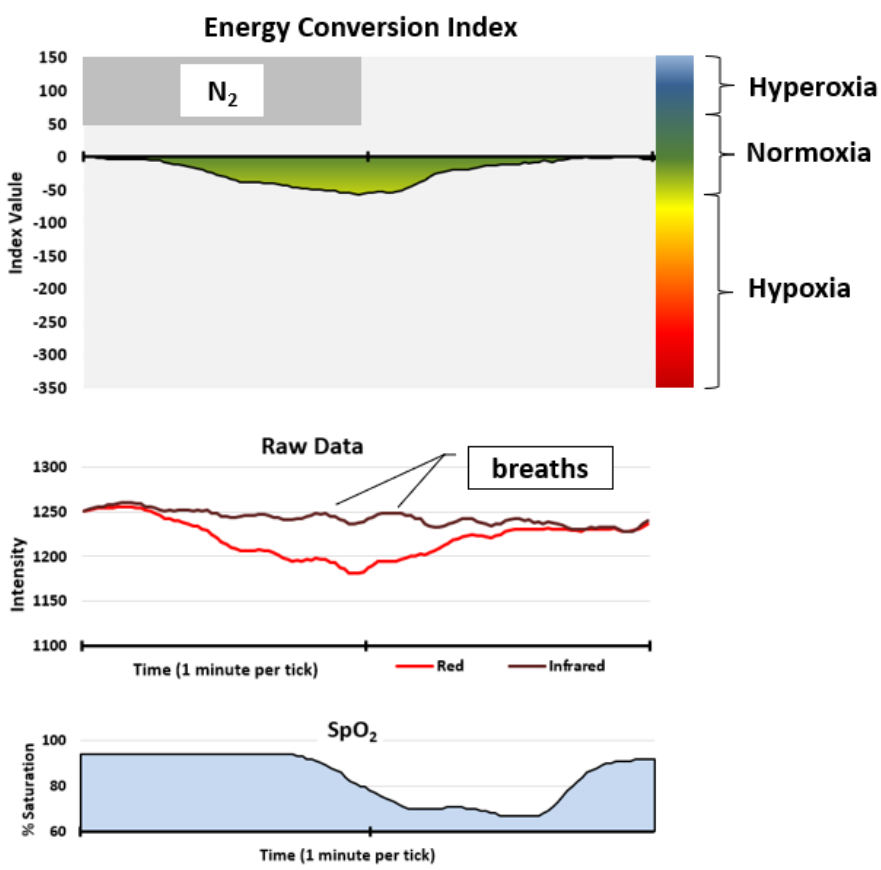

Figure 2. Energy Conversion Index recording while breathing $100 \%$ oxygen $\left(\mathrm{O}_{2}\right)$ for 1 minute.

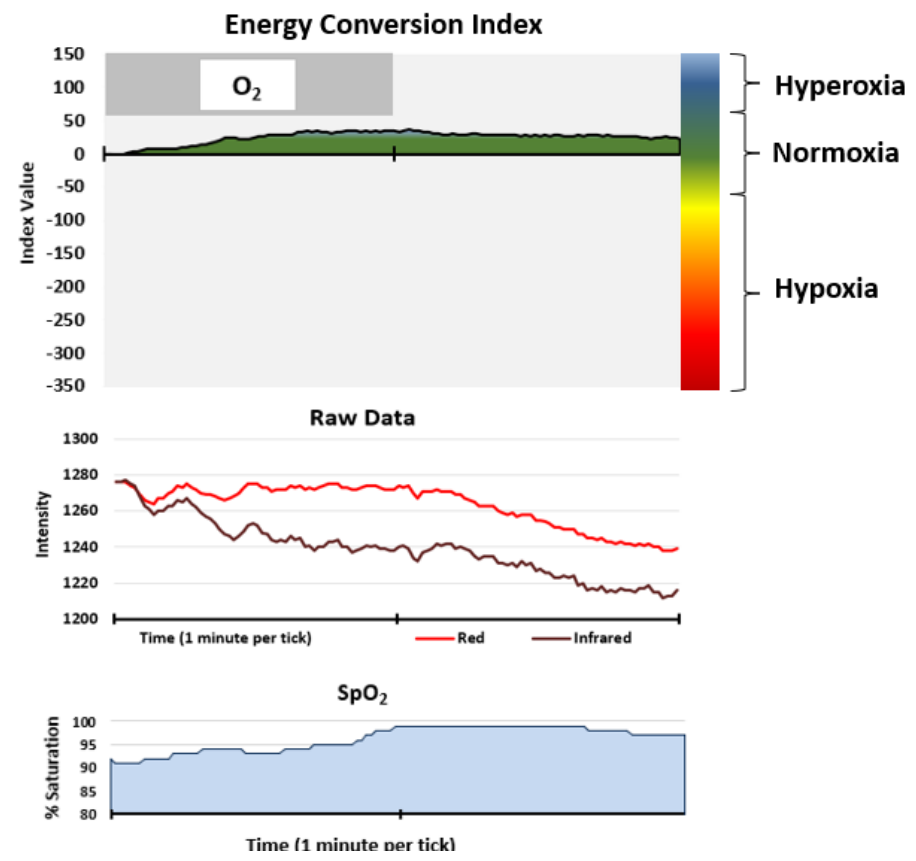


Figure 3. Simultaneous recording during exercise: Energy Conversion Index and the Raw Data from which it is derived, PPG Heart Rate, and $\mathrm{SpO}_{2}$.

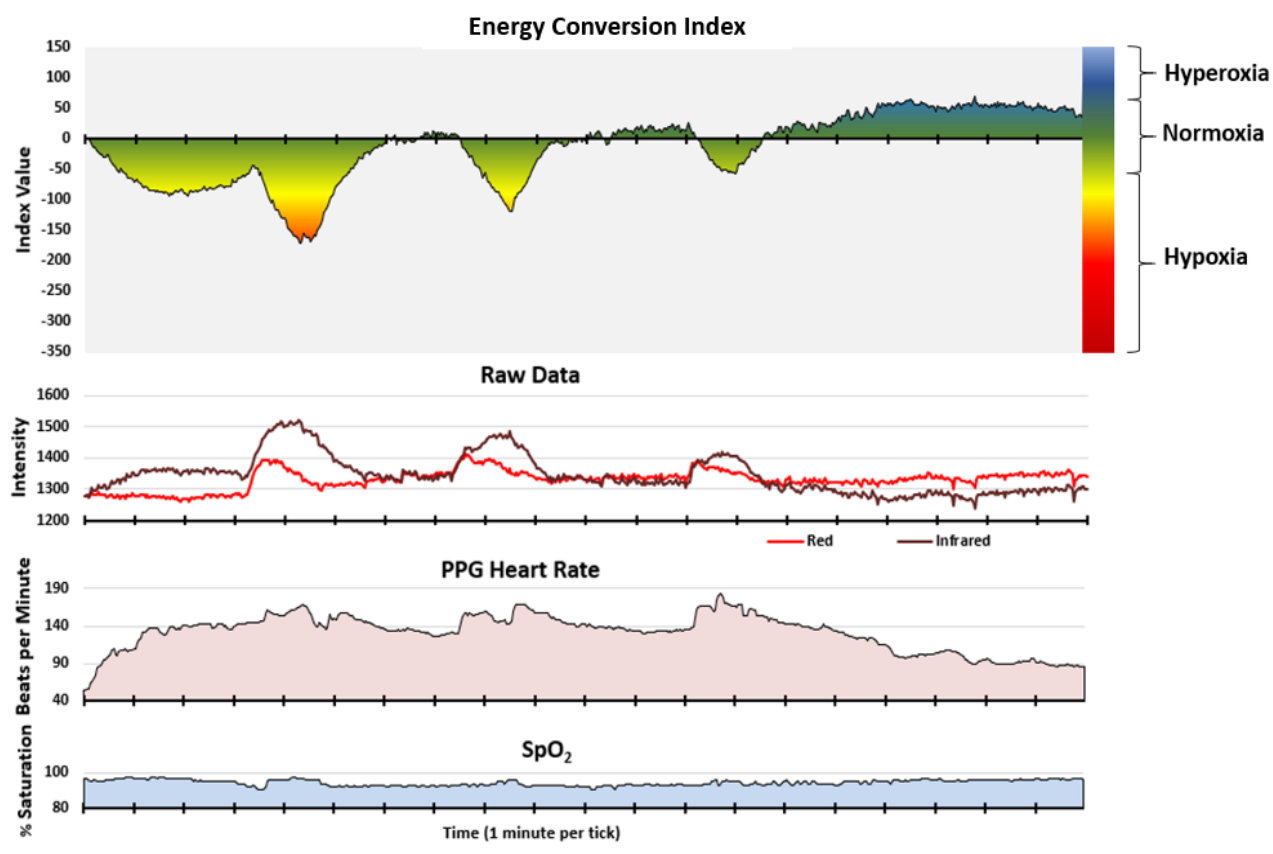




\section{Figure 4. Energy Conversion Index sleep record.}

These three graphs portray the Energy Conversion Index (ECi) signal throughout a sleep study period. The top graph displays the ECi for the entire 9-hour overnight recording. The second graph represents a 10-minute period from this recording, selected based on the subject being asleep; snoring, and without change in $\mathrm{SpO}_{2}$; but with a declining ECi trend. The third graph is a one-minute sample taking from within this segment which corresponds with the raw PSG data appearing in

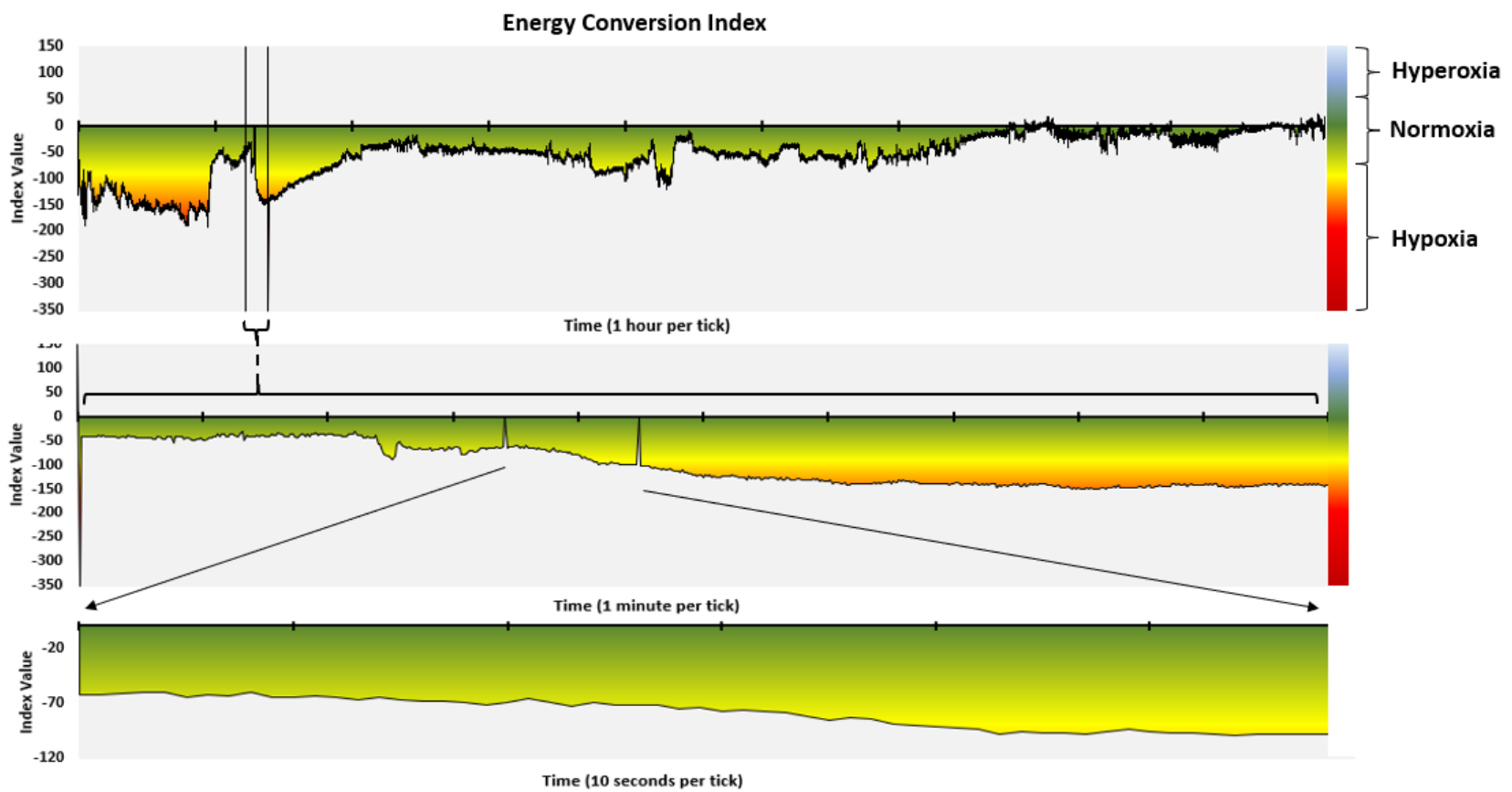


Figure 5. PSG and Energy Conversion Index during primary snoring.

This selected one-minute segment depicts data from a standard PSG consisting of the following channels: Left Outer Canthus of the Eye (LOC); Right Outer Canthus of the Eye (ROC); 6 EEG channels referenced to the contralateral mastoid (F3-M2, F4-M1, C3M2, C4-M1, O1-M2, and O2-M1); 2 Chin EMG Channels; EKG; A Sound Pressure Microphone (Snore); Nasal Pressure (PTAF), Oronasal Thermocouple (Airflow); Inductive Plethysomography Detection of Thoracic and Abdominal Respiratory Effort (Chest and Abdomen); Left and Right Anterior Tibialis EMG (LLeg and RLeg); and Pulse Oximetry (SAO2). The data indicate a decreasing ECi trend during a period that the subject is: asleep; snoring; without pulse oximetry desaturation; without awakening; with only very minimal arousal; and without apnea, hypopnea or obvious hypoventilation.

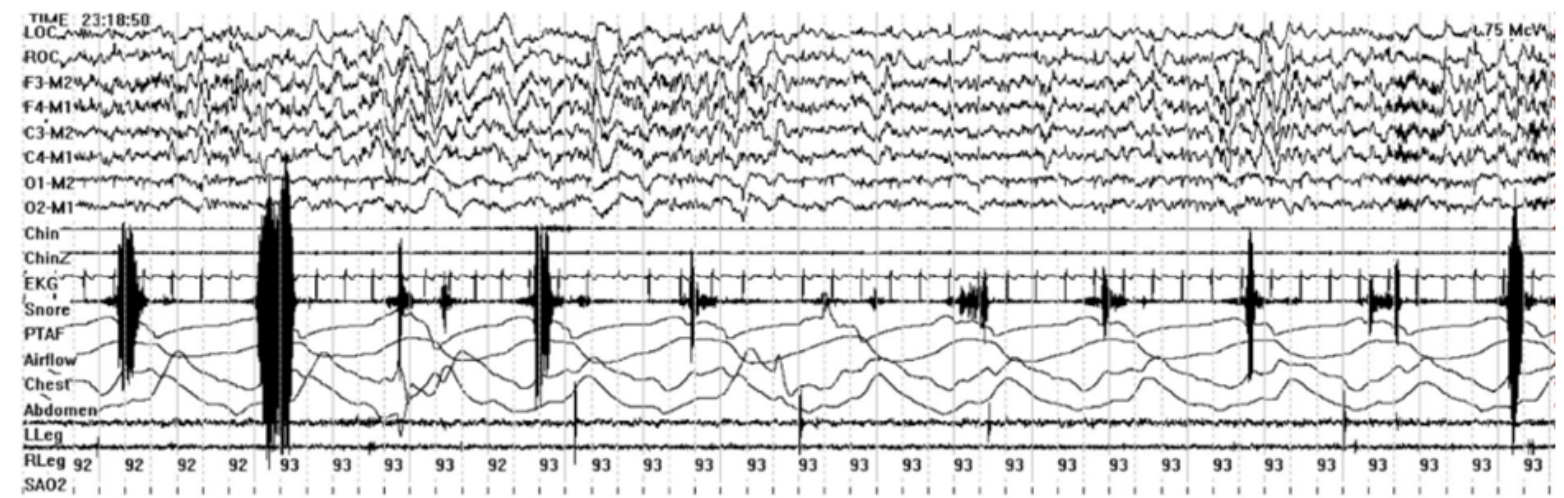

Tissue Oxygen Index

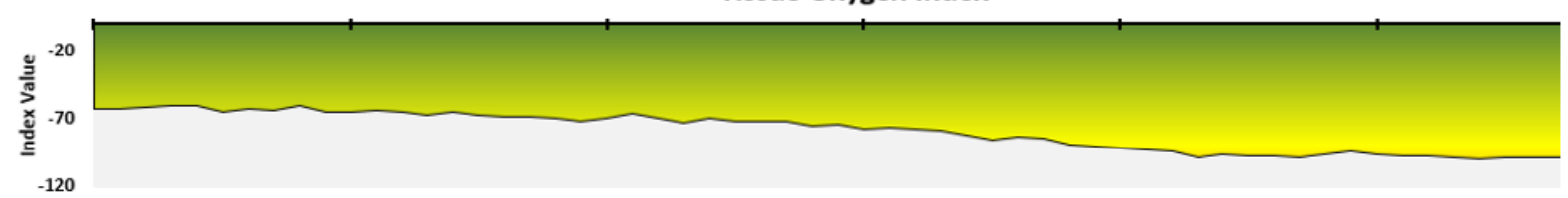

Time (10 seconds per tick) 
Figure 6.a. Apnea Events Accompanied by Drops in ECi

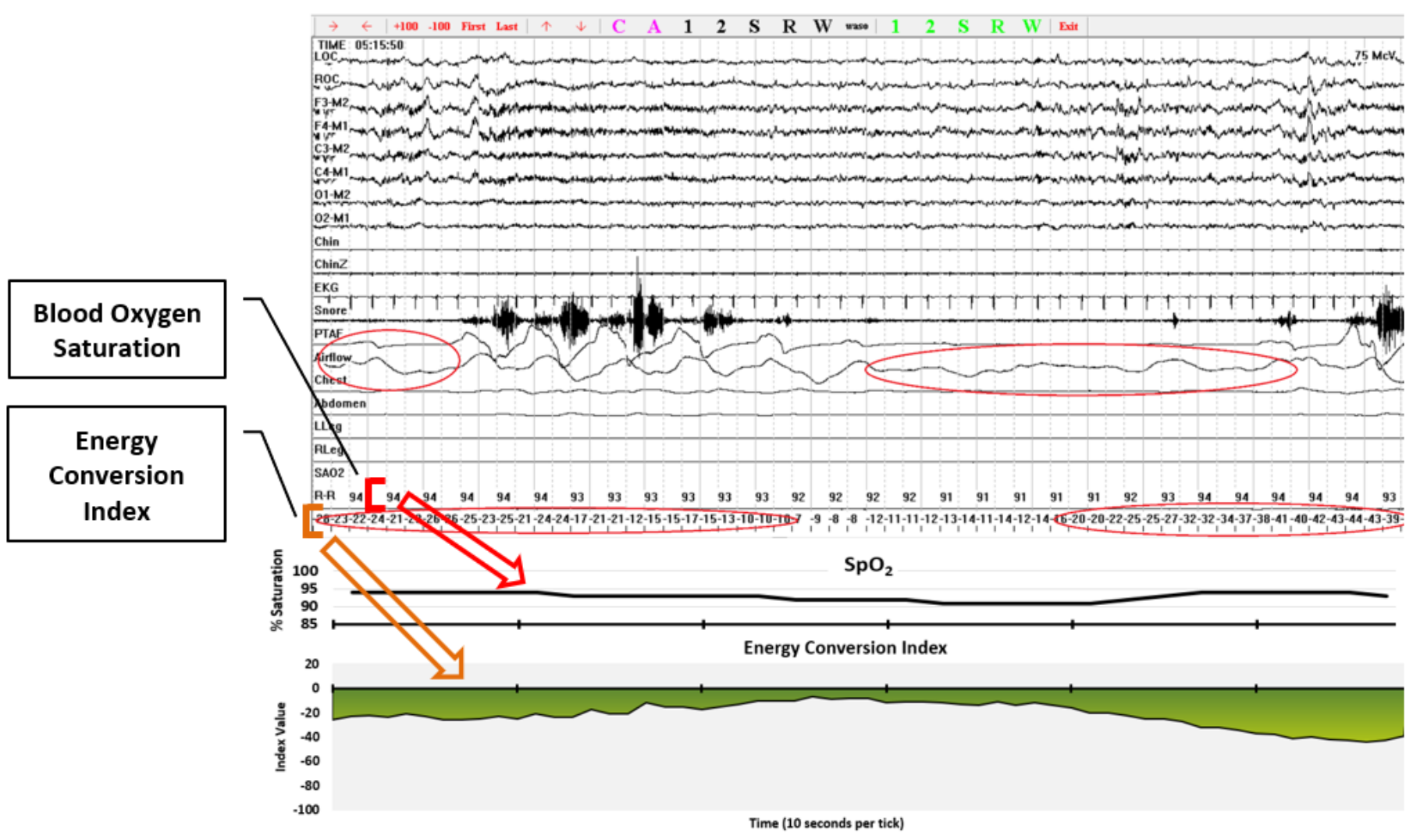


Figure 6.b. Apnea Events Accompanied by Drops in ECi

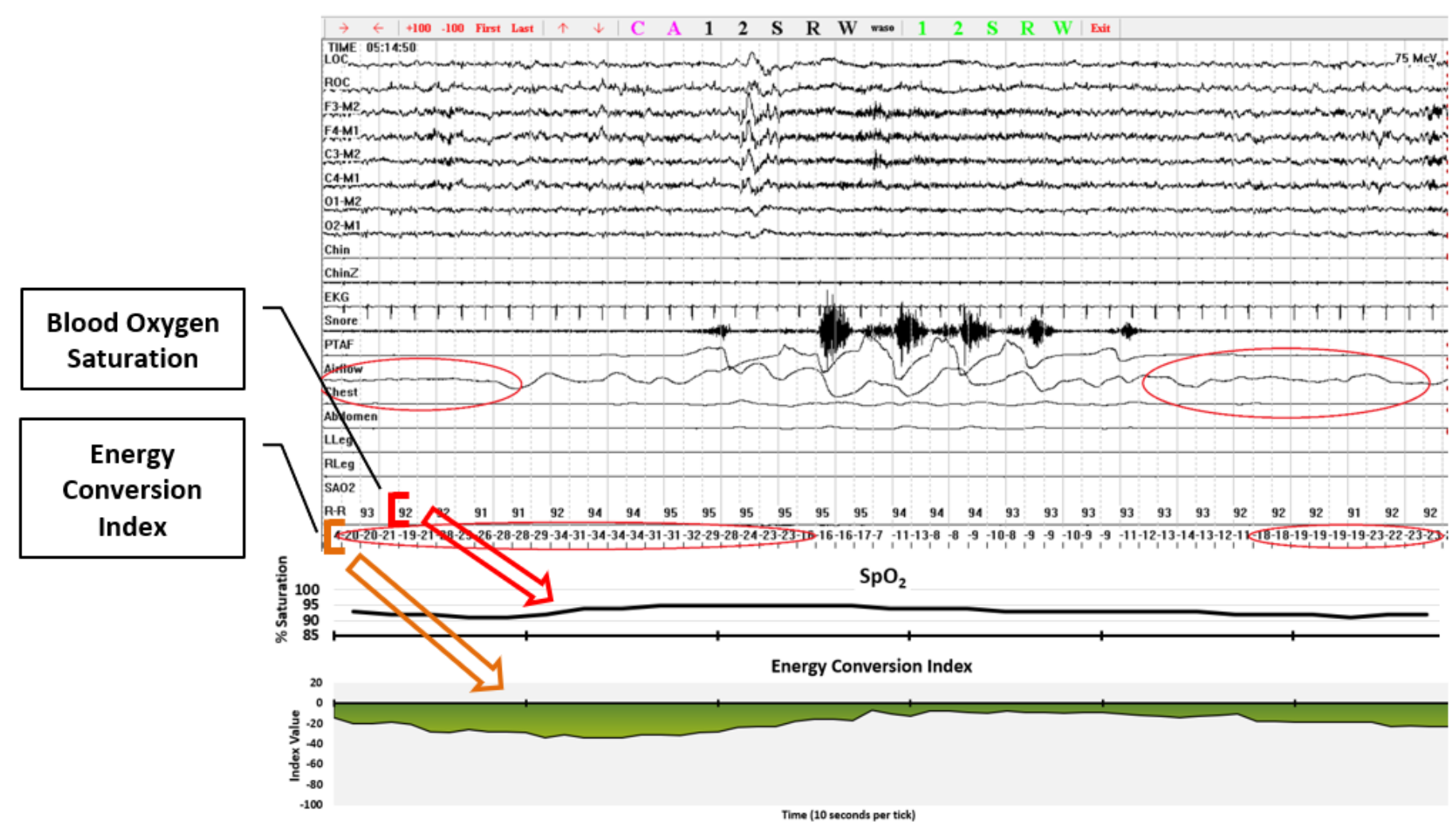

Figure 7.a. Hypoventilation Events (Drops in Nasal Pressure Accompanied by 10 Point Drop in ECi)

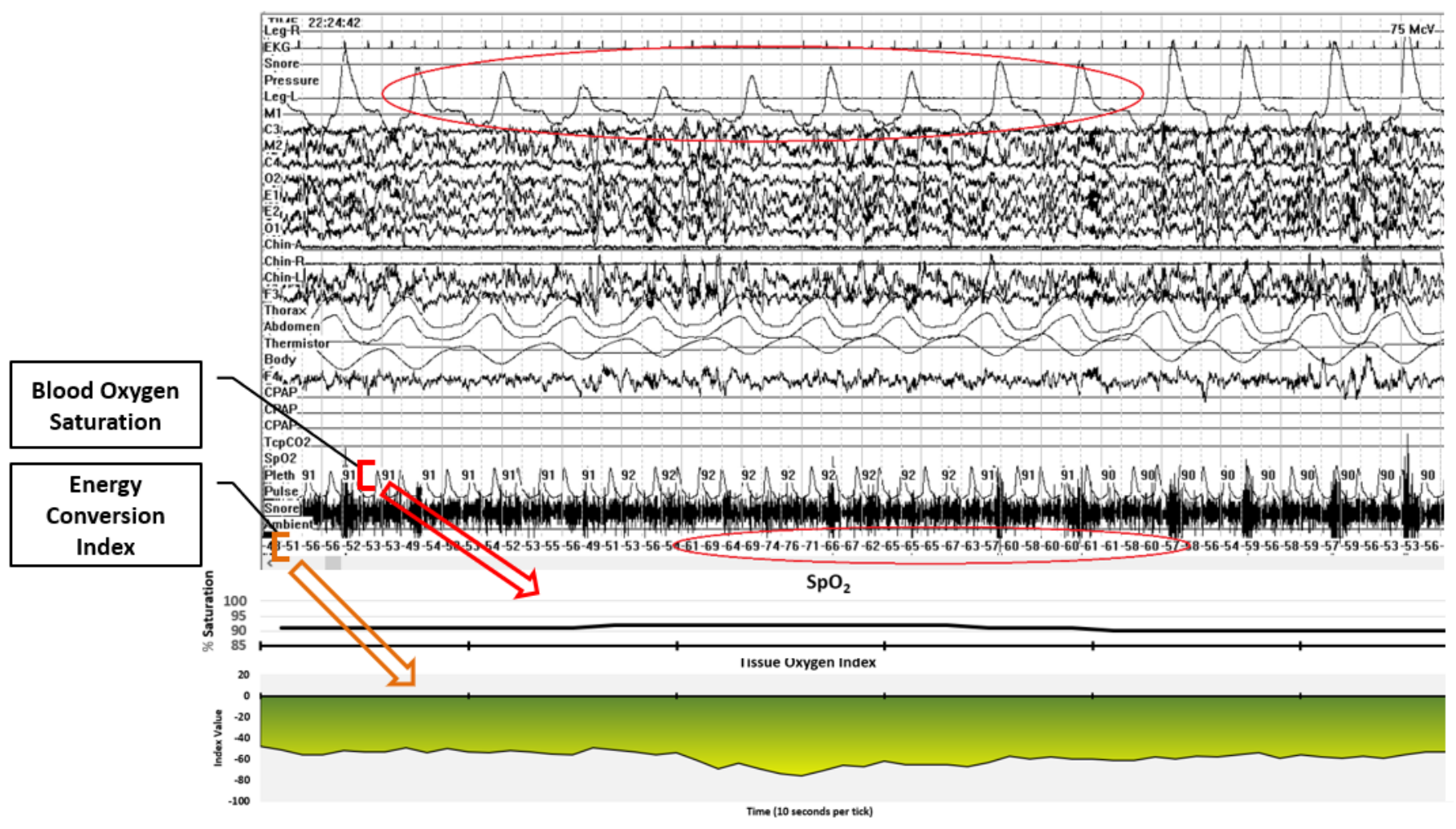

Page 27 of 28 
Figure 7.b. Hypoventilation Events (Drops in Nasal Pressure Accompanied by 10 Point Drop in ECi)

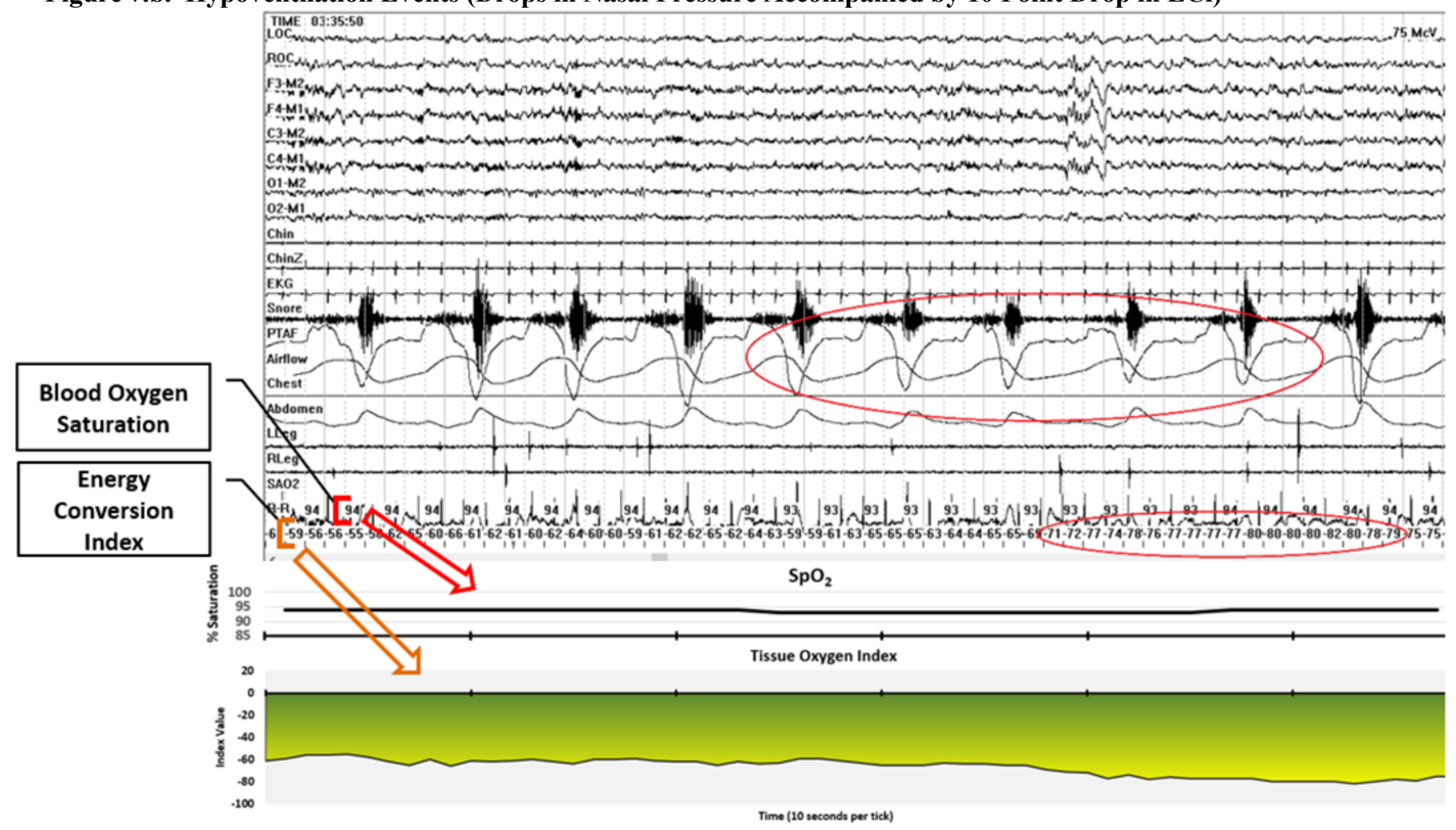

